\title{
A nomogram of preoperative predictors for occult metastasis in patients with PDAC during laparoscopic exploration
}

\author{
Jiachen Ge, Lei Li, Zhaolai Ma, Bin Jiang, Chunhui Yuan, Hangyan Wang, Ying Peng, Dianrong Xiu^ \\ Department of General Surgery, Peking University Third Hospital, Beijing, China \\ Contributions: (I) Conception and design: J Ge, H Wang, D Xiu; (II) Administrative support: Z Ma, C Yuan, D Xiu; (III) Provision of study materials \\ or patients: L Li, B Jiang, Y Peng; (IV) Collection and assembly of data: J Ge, Y Peng; (V) Data analysis and interpretation: J Ge, H Wang, C Yuan; (VI) \\ Manuscript writing: All authors; (VII) Final approval of manuscript: All authors. \\ Correspondence to: Dianrong Xiu. Peking University Third Hospital, 49 North Garden Road, Haidian District, Beijing, China. \\ Email: xiudianrong@foxmail.com.
}

\begin{abstract}
Backgroundk Surgical resection is the only potentially curative treatment for pancreatic ductal adenocarcinoma (PDAC). However, most of patients lose the chance of surgery due to the unresectable disease at the time of diagnosis. Despite the improvement of radiological imaging, a portion of patients intended for radical resection were proven to be unresectable at surgical exploration due to occult metastasis. Methods: Patients who were aimed to undergo radical pancreatectomy for PDAC from 2010 to 2019 were reviewed retrospectively. All patients included underwent diagnostic laparoscopic exploration. Patients were divided into two groups depending on whether distant metastasis were encountered during exploration. Univariate and multivariate logistic regression analyses were used to identify risk factors for occult metastasis. A nomogram to predict occult metastasis of PDAC on exploration was developed and evaluated.

Results: A total of 273 patients who underwent diagnostic laparoscopic exploration were included in this study. Nineteen $(7.0 \%)$ patients were found with distant metastasis during exploration. Multivariate logistic regression analysis showed that ALT>40U/L, CA19-9, CA125 and regional nodes enlargement were independent predictors for occult metastasis. Incorporating these four factors, the nomogram achieved concordance index of 0.799 , with a well-fitted calibration curve.

Conclusions: Occult metastasis is not unusual during surgical exploration in patients with resectable or borderline resectable PDAC. The nomogram could achieve a personal prediction of unexpected distant metastasis on exploration. It may help to sift through patients with PDAC who would benefit from laparoscopic exploration.
\end{abstract}

Keywords: Pancreatic ductal adenocarcinoma (PDAC); occult metastasis; nomogram; laparoscopic exploration; risk factors

Submitted Jul 13, 2020. Accepted for publication Nov 17, 2020.

doi: $10.21037 / g s-20-605$

View this article at: http://dx.doi.org/10.21037/gs-20-605

\section{Introduction}

Pancreatic ductal adenocarcinoma (PDAC) is one of the most aggressive tumors due to its high incidence of locally advanced or metastatic disease. With increasing incidence, it ranks the fourth among the leading causes of cancer- related death in the United States (1). Radical surgical resection combined with systemic therapy remains the only way to gain long-term survival (2). However, only 10-20\% of pancreatic adenocarcinoma are resectable disease at the time of diagnosis (3). And among those anatomic resectable

\footnotetext{
^ ORCID: 0000-0002-9109-3868.
} 
or borderline resectable patients, $5 \%$ to $25 \%$ of patients are found to have occult metastatic disease at exploration (4-6). Although the technology of radiography has been improved over the past few decades, the sensitivity of detecting peritoneal disease and subcentimetric liver metastases is limited (7).

To distinguish PDAC patients at risk of occult metastasis before surgery could avoid unnecessary surgery. Several studies have reported that younger age, male sex, jaundice, weight loss, larger tumor size and higher CA19-9 are predictive factors for occult metastatic disease $(4,8,9)$. Although these results could contribute to estimate the probability of occult metastasis, it still remains far to be clear. Circulating CA125 is often used as a marker to diagnosis ovarian cancer (10). Recently, the roles of CA125 in pancreatic cancer diagnosis and prognosis have been demonstrated. A meta-analysis showed that the CA125based panel is better in diagnosing pancreatic cancer than CA125 or CA19-9 alone (11). Nomogram is a new statistical tool that can help doctors in risk evaluation and clinical decision (12). Therefore, we summarized the experience of one surgical team and performed this study to investigate the links between preoperative predictors, including CA19-9, CA125 and other predictors, and occult metastasis in patients with PDAC at our institution. And then a preoperative predictors based nomogram was constructed and validated to predict the risk of occult metastasis. We present the following article in accordance with the TRIPOD reporting checklist (available at http:// dx.doi.org/10.21037/gs-20-605).

\section{Methods}

\section{Patients selection}

We performed a single-center, retrospective and casecontrol study. The study population was consisted of PDAC patients, who were scheduled to undergo pancreatectomy with curative intent at Peking University Third Hospital between October 2010 and September 2019, including patients with resectable or borderline resectable lesions. Exclusion criteria included patients with local advanced or unresectable disease diagnosed by preoperative imaging, treated with neoadjuvant therapy, with radiographic ascites, with history of other malignant neoplasms within 5 years and with incomplete clinicopathological data $(9,13,14)$. All patients underwent an abdominal and pelvic computed tomography (CT) scan no earlier than 2 weeks before surgery. Magnetic resonance imaging (MRI) scans were usually performed as a supplement when ambiguous lesions were seen on CT and when patients were allergic to iodine contrast agent. All imageological examinations were reviewed by a dedicated radiologist. All procedures were performed in the department of General Surgery, Peking University Third Hospital.

\section{Diagnostic laparoscopy exploration}

In our institution, staging laparoscopy is routinely performed just before radical surgery. The procedure was performed standardized in all patients. Under general anesthesia, a small incision was made in the periumbilical area. Then the Veress needle technique was applied to establish pneumoperitoneum. At $12 \mathrm{mmHg} \mathrm{CO}_{2}$ pneumoperitoneum, a $30^{\circ}$ endoscope was interposed into the trocar and the whole abdominal cavity was inspected with direct visualization, including liver, peritoneum and omentum. Any suspicious lesion was biopsied and sent to frozen section analysis. Based on the outcome, patients were subsequently divided into two groups, with and without occult abdominal metastatic disease. Radical resection was performed when patients without evidence of metastasis after diagnostic laparoscopy exploration. For those patients with frozen pathology confirmed to be occult metastasis, radical operations were terminated and patients might be given palliative treatment according to their main complains.

\section{Data collection}

Preoperative data, including demographic, laboratorial and radiological, were obtained from the institutional database. Operative and pathological data were obtained from electronic medical records. All laboratory tests were no more than 7 days before surgery. Jaundiced patients with cholangitis underwent pre-operative biliary drainage rountinely, and clinical data after then were used. The location of occult metastatic disease was classified into three mutually exclusive groups: liver, peritoneum (including mesentery and omentum) and multiple when metastases were present in two or more locations. The primary outcome was the frequency of occult metastatic disease discovered by laparoscopy. It was defined as the ratio of patients with metastatic disease discovered by exploration and the total number of patients in the study. Then we assessed predictive factors related to a higher risk to find 
occult metastatic disease. The study was conducted in accordance with the Declaration of Helsinki (as revised in 2013). This retrospective study was approved by the Institutional Research Ethics Committee of Peking University Third Hospital (M2020233) and individual consent for this retrospective analysis was waived.

\section{Statistical analysis}

Categorical variables were represented as absolute numbers and percentages and were compared using the $\chi^{2}$ test or Fisher's exact test. For continuous variables, data fitting the normal distribution were displayed as the mean \pm standard deviation, and Student's t test was performed to compare two groups. Nevertheless, data with an abnormal distribution were represented as medians (interquartile ranges), and Mann-Whitney $U$ test was used to compare two groups. Associations between the potential variables and occult metastatic disease were assessed by univariable regression. Variables with a $\mathrm{P}<0.05$ in univariable analysis were included as a covariate in a multivariate logistic regression analysis (forward stepwise logistic regression), in which $\mathrm{P}<0.05$ was deemed as statistically significant. A prediction nomogram was established based on the results of multivariate analysis.

In this study, performance of the nomogram was evaluated by the following indicators. The receiver operating characteristics (ROC) curves was constructed to test the performance of the nomogram and the independent predictive risk factors for occult metastatic disease. The area under the curve (AUC) derived from the ROC curve was calculated respectively and the differences between the nomogram and independent factors were compared. The Youden index were adopted to define the optimal cut-off value. The calibration curve was generated for the evaluation of calibration. Then, decision curve analysis (DCA) was generated to evaluate the nomogram (15). Data analysis and picture plots were performed by SPSS statistical software version 17.0 for Windows (WinWrap Basic) and $\mathrm{R}$ software (version 4.0.2). The differences of AUC between the nomogram and independent factors were calculated by MedCalc (version 19.1.2).

\section{Results}

\section{Patient cobort}

During the study period, a total of 418 patients with pancreatic malignancy underwent staging laparoscopy. 127 patients with other malignancy than PDAC were excluded, including 78 patients with pancreatic neuroendocrine tumor (pNET), 42 patients with intraductal papillary mucinous neoplasm (IPMN), 4 patients with pancreatic metastases and 3 patients with acinic cell adenocarcinoma. A consecutive series of 291 patients with histologically confirmed PDAC underwent laparoscopic surgery. Of them, 18 patients were excluded for various reasons: 6 patients have local advanced disease intended for biopsy or palliative bypass procedure; 11 patients had a history of other malignant tumor within 5 years before operation; 1 patient underwent surgery after liver transplantation. Ultimately, a total of 273 patients were included in this study.

During laparoscopic exploration, 39 patients were detected with suspicious metastatic disease in their abdomen and underwent frozen pathology (Figure 1) (Videos 1 and 2). Apart from 20 patients with benign lesions, 19 (7.0\%) patients were proven to have abdominal occult metastatic disease at surgery, including 9 patients with liver metastasis, 9 patients with peritoneal metastasis, and one patient with both hepatogenic and peritoneal metastases. Among the 19 patients with occult metastasis, 5 patients underwent ${ }^{125} \mathrm{I}$ seeds implantation at the tumor bed; 2 patients underwent biliary-intestinal anastomosis; the other 12 patients just underwent biopsy during operation. After exploration, 254 patients without occult metastatic disease continued to undergo radical surgery. The flow of this study was shown in Figure 2.

\section{Comparisons between patients with and without occult metastasis}

The baseline demographic and clinical characteristics of patients were shown in Table 1. Before exploration, all patients underwent an abdominal and pelvic multidetector computed tomography (MDCT) or MRI. Among the 254 patients without occult metastasis, 250 patients underwent MDCT and 210 patients underwent MRI. All of 19 patients with occult metastasis were performed a MDCT scan and 15 of them underwent MRI. In the term of imaging examination, there was no significant difference between the two groups $(\mathrm{P}=0.999$ and 0.921 for MDCT and MRI, respectively). Between the two groups, there were no significant differences in the fields of age, gender, BMI, abdominal pain, jaundice and history of diabetes mellitus $(\mathrm{P}>0.05)$. Patients with occult metastatic disease were more likely to suffer weight loss $(\mathrm{P}=0.043)$. The ALT 

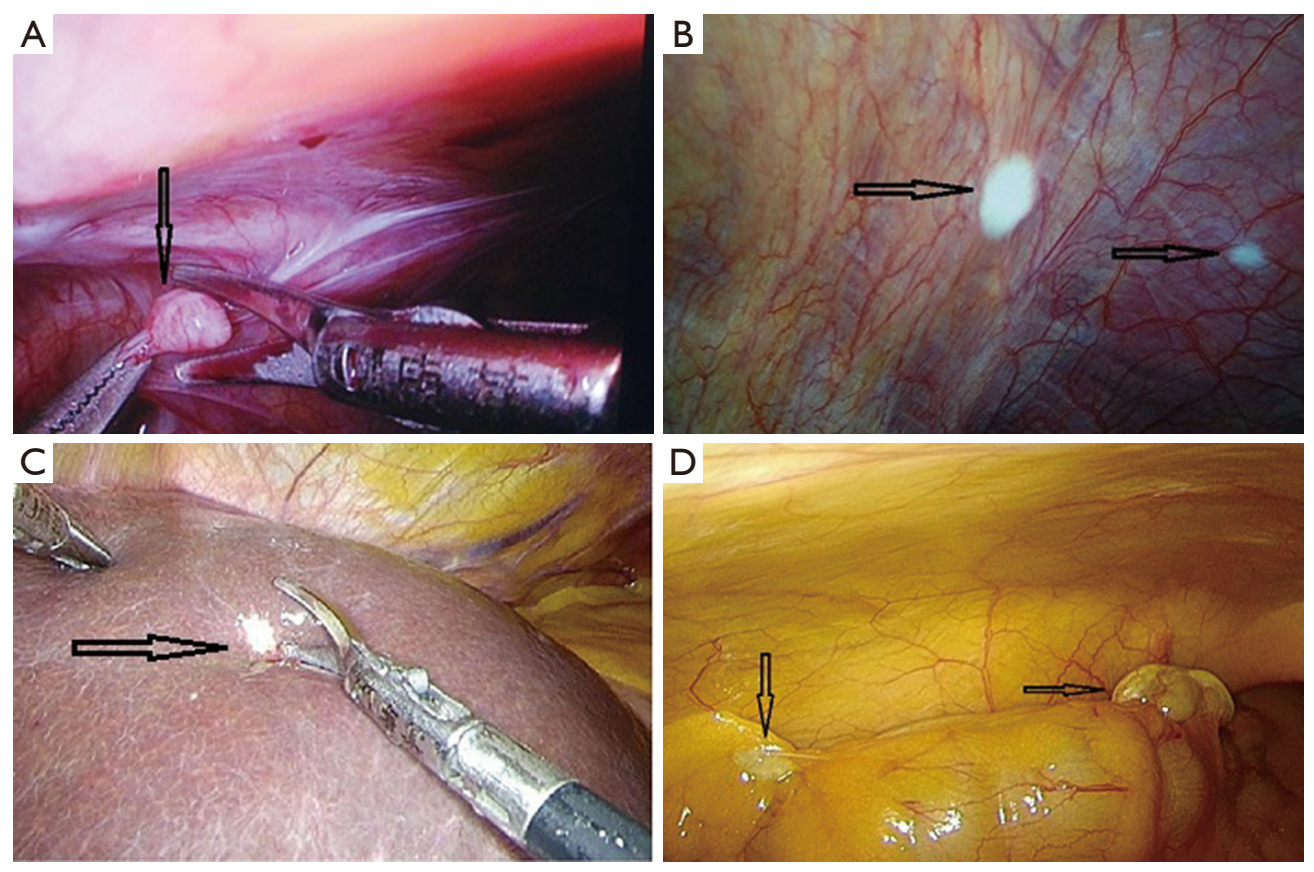

Figure 1 Suspicious metastatic disease (arrow) detected during laparoscopic exploration. (A) Peritoneal metastasis; (B) phrenic metastatic nodules; (C) liver benign nodule; (D) peritoneal benign nodules. These nodules were missed by preoperative imaging check.

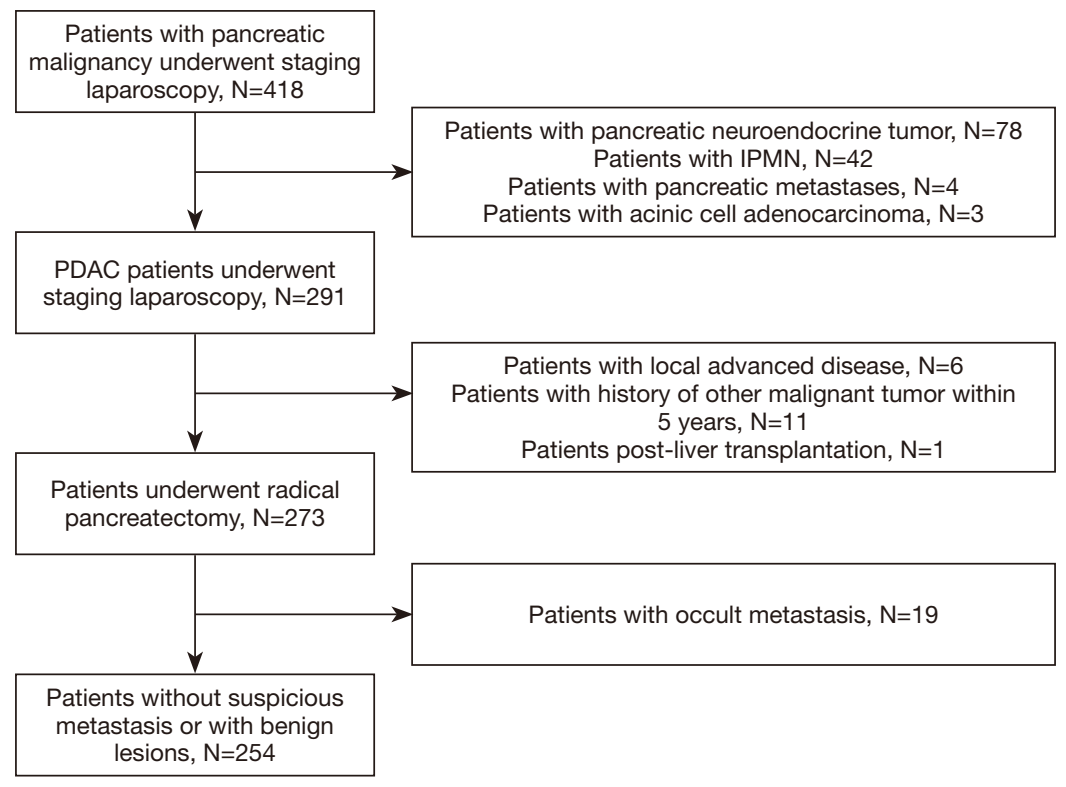

Figure 2 Flow chart of the study. N, number, PDAC, pancreatic ductal adenocarcinoma, IPMN, intraductal papillary mucinous neoplasm.

and AST levels were significantly higher in those without occult metastasis $(\mathrm{P}=0.007$ and 0.009 , respectively). The CEA, CA19-9 and CA125 levels were significantly higher in patients with occult metastatic disease $(\mathrm{P}=0.005,0.020$ and 0.002 , respectively). No significant differences in other laboratory tests included in our study were seen between the two groups. Patients with occult metastasis tended to have lager tumor size and regional lymph nodes enlargement 
Table 1 Comparisons of demographic and clinical factors between patients with and without occult metastases

\begin{tabular}{|c|c|c|c|c|}
\hline & All patients, $n=273$ & Occult metastasis, $n=19$ & $\begin{array}{l}\text { Non-occult metastasis, } \\
\qquad \mathrm{n}=254\end{array}$ & $P$ value \\
\hline Age & $63.00(57.00-70.00)$ & $64.00(61.00-68.50)$ & $63.00(57.00-70.00)$ & 0.561 \\
\hline $\mathrm{BMI}, \mathrm{kg} / \mathrm{m}^{2}$ & $23.15 \pm 3.21$ & $22.31 \pm 0.73$ & $23.22 \pm 3.21$ & 0.236 \\
\hline Abdominal pain & $170(62.3 \%)$ & $15(78.9 \%)$ & $155(61.0 \%)$ & 0.120 \\
\hline Weight loss & $141(51.6 \%)$ & $14(73.7 \%)$ & $127(50.0 \%)$ & 0.046 \\
\hline Diabetes mellius & $81(29.7 \%)$ & $6(31.6 \%)$ & 75 (29.5\%) & 0.850 \\
\hline $\mathrm{Hb}, \mathrm{g} / \mathrm{L}$ & $130.51 \pm 16.79$ & $127.21 \pm 21.73$ & $130.75 \pm 16.39$ & 0.376 \\
\hline ALT, U/L & $38.00(14.00,188.00)$ & $17.00(9.00,62.00)$ & $43.00(15.00,207.75)$ & $0.007^{*}$ \\
\hline AST, U/L & $32.00(17.00,105.00)$ & $18.00(16.00,49.00)$ & $33.00(17.00,124.00)$ & $0.009^{\star}$ \\
\hline TBil, $\mu \mathrm{mol} / \mathrm{L}$ & $18.30(12.50,179.05)$ & $15.60(12.40,24.10)$ & $18.90(12.55,181.55)$ & 0.467 \\
\hline ALP, U/L & $103.00(61.50,330.00)$ & $81.00(66.00,115.00)$ & $108.50(60.75,346.00)$ & 0.311 \\
\hline$y-G T, U / L$ & $57.00(20.50,583.50)$ & $26.00(18.00,66.00)$ & $69.00(21.00,617.25)$ & 0.055 \\
\hline ALB, $g / L$ & $40.76 \pm 4.70$ & $40.72 \pm 4.59$ & $40.77 \pm 4.72$ & 0.968 \\
\hline CEA, ng/mL & $3.47(1.91,6.30)$ & $6.14(3.01,9.89)$ & $3.37(1.87,5.70)$ & $0.005^{\star}$ \\
\hline $\begin{array}{l}\text { Resectability (resectable vs. Borderline } \\
\text { resectable) }\end{array}$ & $182(66.7 \%) / 91(33.3 \%)$ & $11(57.9 \%) / 8(42.1 \%)$ & $171(67.3 \%) / 83(32.7 \%)$ & 0.400 \\
\hline Primary tumor size on MDCT or MRI, mm & $30.00(22.00,39.50)$ & $39.00(33.00,58.00)$ & $28.75(22.00,39.00)$ & $0.001^{*}$ \\
\hline $\begin{array}{l}\text { Regional lymph nodes enlargement on } \\
\text { imaging, } \mathrm{n}(\%)\end{array}$ & $75(27.5 \%)$ & $10(52.6 \%)$ & $65(25.6 \%)$ & $0.011^{\star}$ \\
\hline
\end{tabular}

n, number; BMI, body mass index; Hb, hemoglobin; Neu/Lym, neutrophil-to-lymphocyte ratio; Lym/Mono, lymphocyte-to-monocyte ratio; PIt/Lym, platelet-to-lymphocyte ratio; ALT, alanine aminotransferase; AST, aspartate transaminase; TBIL, total bilirubin; ALP, alkaline phosphatase; y-GT, gamma-glutamyltransferase; ALB, albumin; CEA, carcinoembryonic antigen; MDCT, multi-detector computed tomography; MRI, magnetic resonance imaging. * $\mathrm{P}<0.05$.

on imaging $(\mathrm{P}=0.001$ and 0.011 , respectively). Moreover, patients with occult metastases had a lower y-GT level, and were incline to be located at body or tail, though not significant $(\mathrm{P}=0.055$ and 0.096 , respectively).
To further elucidate the independent predictors for occult metastatic disease, multivariate logistic analysis was performed. The weight loss or not, ALT $>40 \mathrm{U} / \mathrm{L}, \mathrm{CEA}$, CA19-9, CA125, tumor location, tumor size $>40 \mathrm{~mm}$ and 
Table 2 Multivariate regression results of predictors for occult metastases

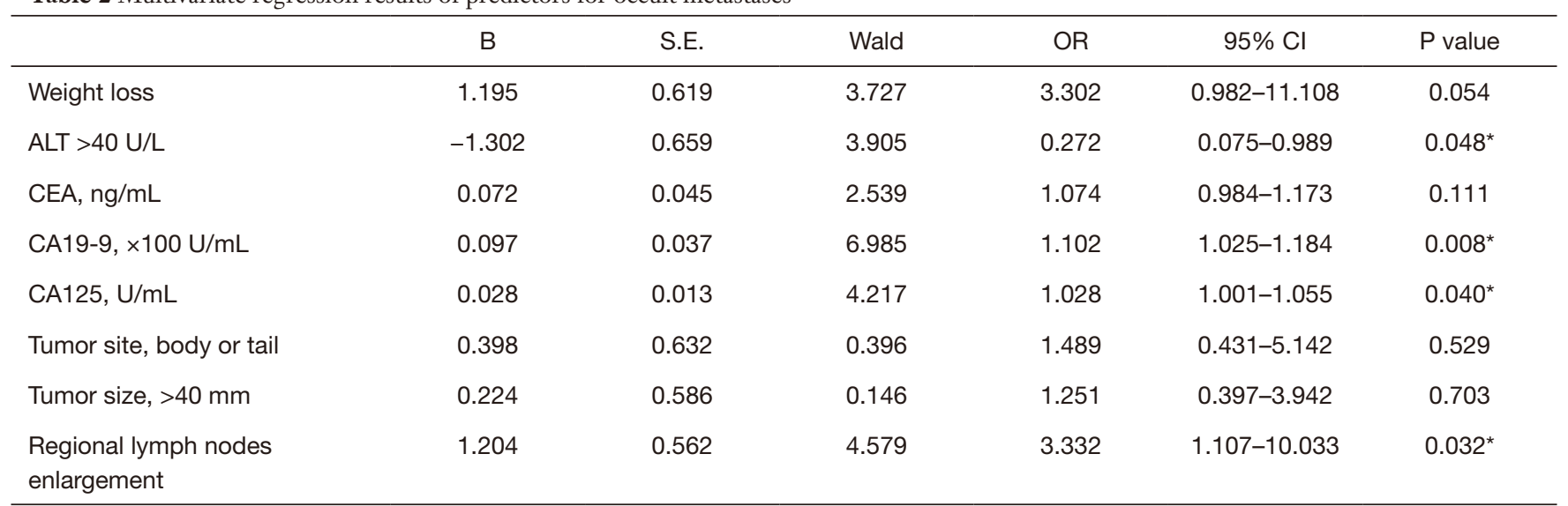

B, regression coefficient; S.E., standard error; OR, odds ratio; $\mathrm{Cl}$, confidence interval; ALT, alanine aminotransferase. * $\mathrm{P}<0.05$.

regional lymph nodes enlargement were included so that the analysis was more clinical relevant. The results showed that ALT >40 U/L, CA19-9, CA125 and regional lymph nodes enlargement were independent predictors of occult metastatic disease. With one hundred one-units increase of CA19-9, the probability of occult metastasis increased by $10.2 \%(\mathrm{OR}=1.102 ; 95 \% \mathrm{CI}, 1.025-1.184 ; \mathrm{P}=0.008)$. And the probability of occult metastasis increased by $2.8 \%$ (OR $=1.028 ; 95 \%$ CI, 1.001-1.055; P=0.040) with a one-unit increase of CA125 (Table 2).

\section{Nomogram for predicting occult metastasis}

Based on the independent predictors for occult metastasis, a nomogram was created (Figure 3). The AUC of the nomogram was 0.799 (95\% CI: 0.692-0.906). Despite not significantly higher than the AUC of CA125 ( $\mathrm{P}=0.185)$, the AUC of the nomogram was significantly higher than that of CA19-9 ( $\mathrm{P}=0.030)$ (Figure 4 and Table 3). The calibration curve indicated that the prediction of the nomogram agreed well with actual observations (Figure 5). The DCA model showed that with a threshold probability of $5 \%$ to $65 \%$, the nomogram could predict occult metastasis than "treat all" or "treat non" schemes (Figure 6). And the nomogram does a better job in predicting occult metastasis than CA125 or CA19-9 alone.

\section{Discussion}

In this study, we retrospectively summarized patients who underwent surgical exploration for resectable or borderline resectable PDAC in our center in order to investigate the preoperative risk factors for occult metastasis. A nomogram combining the predictors was developed. To our knowledge, this is the first nomogram to predict occult metastatic disease for patients with PDAC, providing a method to measure the risk of individual patients. Nowadays radical surgery is regarded as the only potentially curative treatment for PDAC. Accurate determination of resectability in patients with PDAC remains challenging despite the advanced radiological technology (16). Nevertheless, occult metastatic disease from PDAC is not unusual during surgery. Previous studies have shown that about $8-26 \%$ patients with resectable PDAC staged by MD-CT were proven to have metastasis during exploration $(4,6,9)$. In consideration of the high peri-operative mortality and morbidity of pancreatic surgery, non-curative exploratory laparotomy of pancreas should be avoided. Najah et al. indicated that single-incision laparoscopic peritoneal exploration could be safely applied in the management of patients with peritoneal metastases (17). Several studies have concluded that compared to laparotomy, diagnostic laparoscopy routinely used not only saves patients an incision, but also shortens the intervals between operation and adjuvant therapy (18-20). However, given the high cost, low proportion of metastasis and limited additional value, it is still controversial whether staging laparoscopy should be applied routinely or selectively (21). A cost analysis indicated that the case-specific or routine use of laparoscopy in pancreatic cancer did not add significantly to the overall expense of treatment (22). Another study concluded that staging laparoscopy became cost-effective by diverting unresectable patients from operative to 


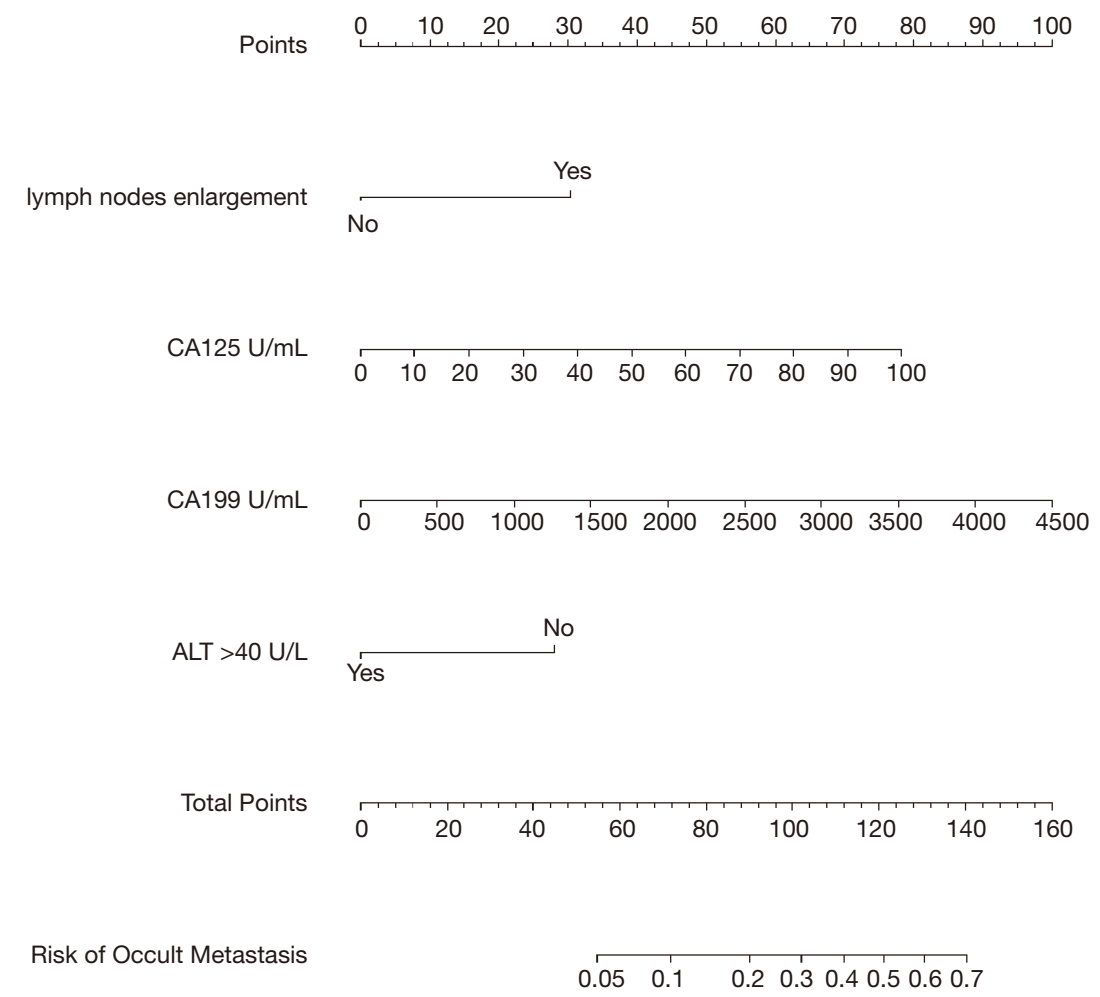

Figure 3 Nomogram for predicting occult metastasis during laparoscopic exploration. To estimate the risk of occult metastasis, mark patients value at each axis, then draw a straight line perpendicular to the point axis. After sum the points of all variables, mark the sum on the total points axis and make a vertical line to the risk axis. ALT, alanine aminotransferase.

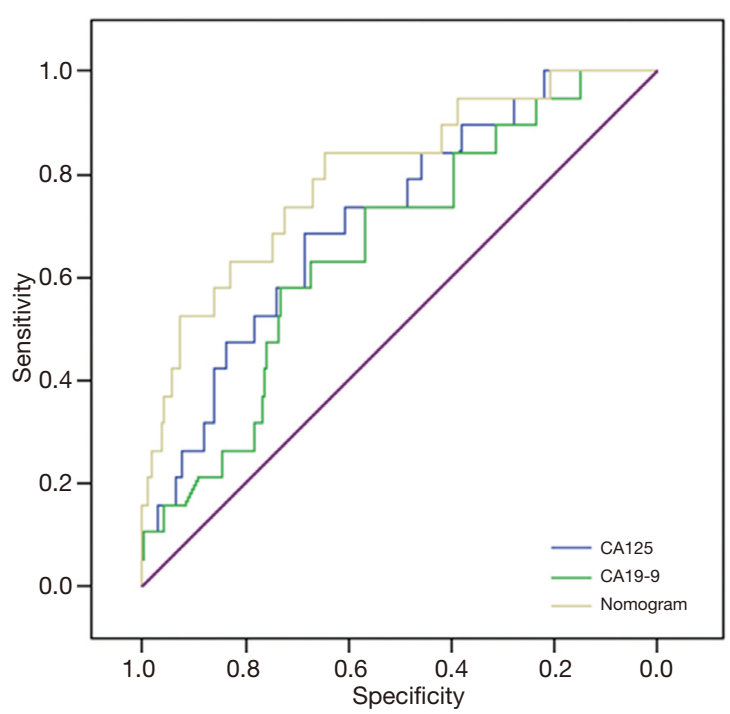

Figure 4 Receiver operating characteristic (ROC) curves of the nomogram, CA125 and CA19-9. endoscopic palliation (23). However, in the same study, given the paucity of missed metastases on MRI, the yield of staging laparoscopy was marginal and its cost-effectiveness was poor. Therefore, selecting patients who were highly likely to be with occult metastases could increase the cost-effectiveness of diagnostic laparoscopy. In this study, only $7 \%$ patients were found with distant metastasis during exploration, so the cost-effectiveness seemed to be even lower. This may be owing to the fact that most of patients in this study underwent MRI pre-operation, which was superior to MD-CT in detecting small liver metastases (24). What's more, we should add the fact that laparoscopy exploration cost patients less than 300 American dollars in China. On account of the relatively low cost, routine laparoscopic exploration was done for most of patients with abdominal neoplasms since 2003 (25).

A couple of factors have been reported to be associated with occult metastatic disease, including younger age, 
Table 3 Comparison of AUCs among predictors for occult metastasis

\begin{tabular}{|c|c|c|c|c|c|}
\hline Predictors & AUC & $95 \% \mathrm{Cl}$ of $\mathrm{AUC}$ & Cut-off & Sensitivity & Specificity \\
\hline CA125 & 0.715 & $0.603-0.828$ & 23 & 0.684 & 0.685 \\
\hline CA19-9 & 0.660 & $0.542-0.778$ & 472 & 0.579 & 0.732 \\
\hline
\end{tabular}

AUC, area under the curve; $\mathrm{Cl}$, confidence interval.

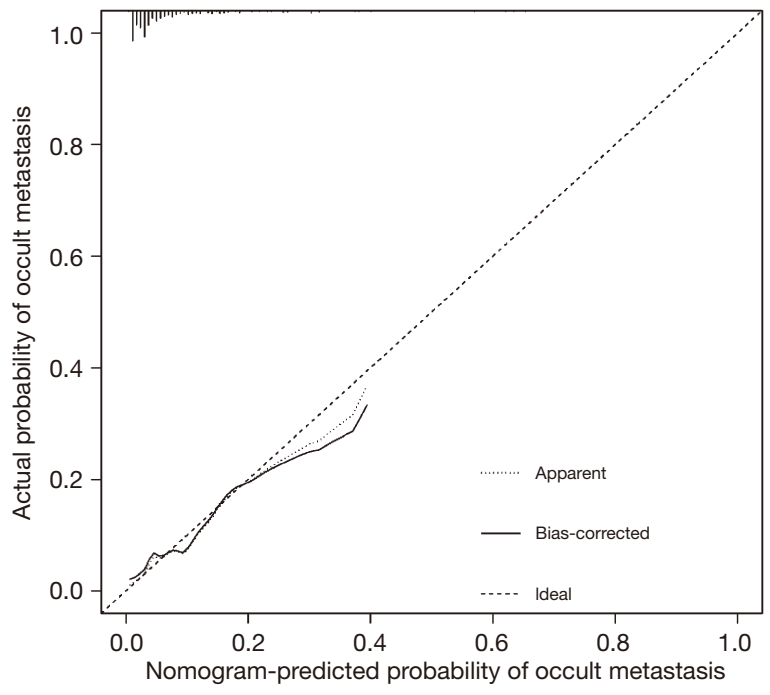

Figure 5 Calibration curve for nomogram. The curve depicts the calibration of the nomogram in terms of agreement between predicted and actual probability of occult metastasis.

male sex, weight loss, larger tumor size, higher CA19-9, suspicious lymph node and more extensive peripancreatic tumor infiltration et al. $(4,6,9,26)$. Our study showed that ALT $>40$ U/L, CA19-9, CA125 and regional nodes enlargement were independent predictors for occult metastasis. Moreover, the nomogram based on the predictors was established. CA19-9 is the most commonly used and best validated serum tumor marker for diagnosis and monitoring therapy in patients with PDAC (27). A number of studies have reported that CA19-9 level is associated with tumor burden and advanced stages (28-30). Though significantly affected by bilirubin level, CA19-9 has been proven to be a good predictor of unresectability even in patients with jaundice. Regional lymph node status is also regarded as one of the most prognostic indicators in patients with PDAC $(31,32)$. In this study, weight loss, CEA level and tumor size were significantly associated with distant metastasis in univariate analysis, but not

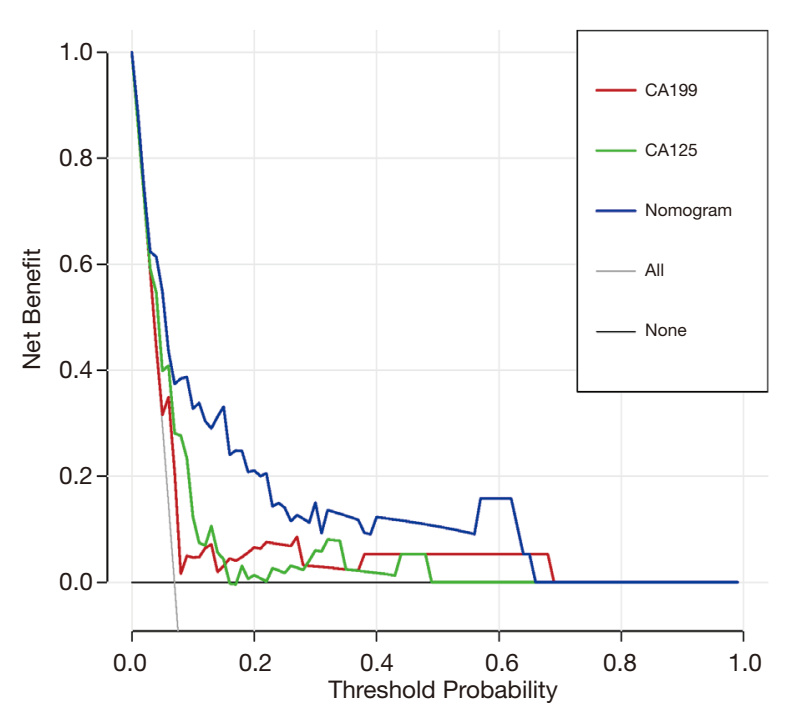

Figure 6 The decision curve analysis (DCA) for the nomogram. The net benefit was plotted versus the threshold probability. The blue, red and green line represents the nomogram, CA19-9 and CA125 respectively. The gray line represents the treat-all-patients scheme and the black line represents the treat-none scheme.

significant in multivariate analysis. Age and gender were not predictors, which might be explained by the small sample size of this study. Lower ALT and AST levels were found to be associated with occult metastases. As indexes of liver function, ALT and AST levels would elevate if liver metastasis or liver injuries occurred. However, the roles of ALT and AST in the prognosis of PDAC remain unknown currently. In a large multicenter cohort study of ten years, elevated ALT and AST levels predicted a worsened prognosis in patients with stage IV PDAC patients, but not of prognostic value in stage III patients (33). In a recent study, low ALT level was regarded as an independent predictors of unexpected distant metastasis (9). Despite meant normal liver function, patients with lower ALT and AST levels are more likely to be asymptomatic, which may delay their diagnosis. Our study also showed that higher 
CA125 level was a risk factor of occult metastasis. CA125, which is encoded by the MUC16 gene, is often used as a marker to diagnosis ovarian cancer. And the roles of CA125 in pancreatic cancer diagnosis and prognosis have been demonstrated recently (34-36). As a tumor marker not related to serum bilirubin levels, CA125 might be superior to CA19-9 in the diagnosis of pancreatic cancer patients with jaundice. Liu et al. showed that among the frequentlyused tumor markers, serums CA 125 was the most strongly associated with pancreatic cancer metastasis (37). CA125 levels increased with increasing metastasis to lymph nodes and distant organs, especially the liver. Our results confirmed that high serum CA125 level predicted unresectable pancreatic cancer due to the occult metastases.

Nowadays, many pancreatic surgeons hold a view that the high rate of early recurrence and poor prognosis of PDAC is attributed to the presence of occult metastases at the time of resection (38). Accurate staging pre-operation is worthwhile for surgeons and radiologists to dedicate more. Although somewhat controversial, positive peritoneal lavage cytology obtained at laparoscopy or laparotomy is seen as equivalent to M1 disease (39). If resection has been done in such a patient, he or she should be treated for M1 disease. The study of Harada et al. confirmed that 5-ALA-PDD held promise for rapid diagnosis of peritoneal metastasis in patients with pancreatic cancer (40). The new imaging technique, which helps to the diagnosis of metastases, remains to be studied.

There are several limitations associated with this study that should be noted. First, as a retrospective study in a single institution, the sample size was relatively small, and there was a potential for several biases. For example, age and gender were not predictors in this study. Second, despite the good performance in AUC, calibration curve and DCA, the nomogram needs further independent validation. Finally, we did not include patients who underwent neoadjuvant therapy, because the tumor parameters included in this study would be changed greatly after it.

\section{Conclusions}

In conclusion, we retrospected cases of PDAC at our institution in recent years and selected preoperative risk factors for occult metastasis. A nomogram was constructed for predicting the risk of occult metastasis visually. It may help physicians to sift through patients with PDAC who would benefit from laparoscopic exploration, resulting in more cost-effective investigations.

\section{Acknowledgments}

Authors would like to thank Professor Yi-Mu Jia who provided photographs taken during surgery.

Funding: This study was supported by a grant from the Capital Medical Development Research Foundation Project (No. 2018-1-4021).

\section{Footnote}

Reporting Checklist: The authors have completed the TRIPOD reporting checklist. Available at http://dx.doi. org/10.21037/gs-20-605

Data Sharing Statement: Available at http://dx.doi. org/10.21037/gs-20-605

Conflicts of Interest: All authors have completed the ICMJE uniform disclosure form (available at http://dx.doi. org/10.21037/gs-20-605). The authors have no conflicts of interest to declare.

Ethical Statement: The authors are accountable for all aspects of the work in ensuring that questions related to the accuracy or integrity of any part of the work are appropriately investigated and resolved. The study was conducted in accordance with the Declaration of Helsinki (as revised in 2013). This study was approved by Institutional Review Board of Peking University Third Hospital (M2020233) and individual consent for this retrospective analysis was waived.

Open Access Statement: This is an Open Access article distributed in accordance with the Creative Commons Attribution-NonCommercial-NoDerivs 4.0 International License (CC BY-NC-ND 4.0), which permits the noncommercial replication and distribution of the article with the strict proviso that no changes or edits are made and the original work is properly cited (including links to both the formal publication through the relevant DOI and the license). See: https://creativecommons.org/licenses/by-nc-nd/4.0/.

\section{References}

1. Zhu H, Li T, Du Y, et al. Pancreatic cancer: challenges and opportunities. BMC Med 2018;16:214.

2. Schnelldorfer T, Ware AL, Sarr MG, et al. Longterm survival after pancreatoduodenectomy for 
pancreatic adenocarcinoma: is cure possible. Ann Surg 2008;247:456-62.

3. Kleeff J, Korc M, Apte M, et al. Pancreatic cancer. Nat Rev Dis Primers 2016;2:16022.

4. Slaar A, Eshuis WJ, van der Gaag NA, et al. Predicting distant metastasis in patients with suspected pancreatic and periampullary tumors for selective use of staging laparoscopy. World J Surg 2011;35:2528-34.

5. Allen VB, Gurusamy KS, Takwoingi Y, et al. Diagnostic accuracy of laparoscopy following computed tomography (CT) scanning for assessing the resectability with curative intent in pancreatic and periampullary cancer. Cochrane Database Syst Rev 2016;7:CD009323.

6. Gemenetzis G, Groot VP, Blair AB, et al. Incidence and risk factors for abdominal occult metastatic disease in patients with pancreatic adenocarcinoma. J Surg Oncol 2018;118:1277-84.

7. Shirakawa S, Toyama H, Kido M, et al. A prospective single-center protocol for using near-infrared fluorescence imaging with indocyanine green during staging laparoscopy to detect small metastasis from pancreatic cancer. BMC Surg 2019;19:165.

8. Maithel SK, Maloney S, Winston C, et al. Preoperative CA 19-9 and the yield of staging laparoscopy in patients with radiographically resectable pancreatic adenocarcinoma. Ann Surg Oncol 2008;15:3512-20.

9. Liu X, Fu Y, Chen Q, et al. Predictors of distant metastasis on exploration in patients with potentially resectable pancreatic cancer. BMC Gastroenterol 2018;18:168.

10. Diamandis EP, Bast RC, Gold P, et al. Reflection on the discovery of carcinoembryonic antigen, prostate-specific antigen, and cancer antigens CA125 and CA19-9. Clin Chem 2013;59:22-31.

11. Meng Q, Shi S, Liang C, et al. Diagnostic Accuracy of a CA125-Based Biomarker Panel in Patients with Pancreatic Cancer: A Systematic Review and Meta-Analysis. J Cancer 2017;8:3615-22.

12. Eastham JA, Kattan MW, Scardino PT. Nomograms as predictive models. Semin Urol Oncol 2002;20:108-15.

13. de Jesus VH, da Costa Junior WL, de Miranda Marques $T$, et al. Role of staging laparoscopy in the management of Pancreatic Duct Carcinoma (PDAC): Single-center experience from a tertiary hospital in Brazil. J Surg Oncol 2018;117:819-28.

14. Takahara N, Isayama H, Nakai Y, et al. Pancreatic cancer with malignant ascites: clinical features and outcomes. Pancreas 2015;44:380-5.

15. Vickers AJ, Elkin EB. Decision curve analysis: a novel method for evaluating prediction models. Med Decis Making 2006;26:565-74.

16. Soloff EV, Zaheer A, Meier J, et al. Staging of pancreatic cancer: resectable, borderline resectable, and unresectable disease. Abdom Radiol (NY) 2018;43:301-13.

17. Najah H, Malgras B, Dohan A, et al. The role of singleincision laparoscopic peritoneal exploration in the management of patients with peritoneal metastases. Surg Endosc 2020;34:2040-9.

18. Fong ZV, Alvino D, Fernández-Del Castillo C, et al. Reappraisal of Staging Laparoscopy for Patients with Pancreatic Adenocarcinoma: A Contemporary Analysis of 1001 Patients. Ann Surg Oncol 2017;24:3203-11.

19. Paracha M, Van Orden K, Patts G, et al. Opportunity Lost? Diagnostic Laparoscopy in Patients with Pancreatic Cancer in the National Surgical Quality Improvement Program Database. World J Surg 2019;43:937-43.

20. Sell NM, Fong ZV, Del Castillo CF, et al. Staging Laparoscopy Not Only Saves Patients an Incision, But May Also Help Them Live Longer. Ann Surg Oncol 2018;25:1009-16.

21. Schnelldorfer T, Gagnon AI, Birkett RT, et al. Staging laparoscopy in pancreatic cancer: a potential role for advanced laparoscopic techniques. J Am Coll Surg 2014;218:1201-6.

22. Enestvedt CK, Mayo SC, Diggs BS, et al. Diagnostic laparoscopy for patients with potentially resectable pancreatic adenocarcinoma: is it cost-effective in the current era. J Gastrointest Surg 2008;12:1177-84.

23. Tapper E, Kalb B, Martin DR, et al. Staging laparoscopy for proximal pancreatic cancer in a magnetic resonance imaging-driven practice: what's it worth. HPB (Oxford) 2011;13:732-7.

24. Tsurusaki M, Numoto I, Oda T, et al. Assessment of Liver Metastases Using CT and MRI Scans in Patients with Pancreatic Ductal Adenocarcinoma: Effects of Observer Experience on Diagnostic Accuracy. Cancers (Basel) 2020;12:1455.

25. Zhang T, Du X, Zhao Y. Laparoscopic surgery for pancreatic lesions: current status and future. Front Med 2011;5:277-82.

26. Lee HS, An C, Hwang HK, et al. Preoperative prediction of futile surgery in patients with radiologically resectable or borderline resectable pancreatic adenocarcinoma. J Gastroenterol Hepatol 2020;35:499-507.

27. Takaori K, Bassi C, Biankin A, et al. International Association of Pancreatology (IAP)/European Pancreatic Club (EPC) consensus review of guidelines 
for the treatment of pancreatic cancer. Pancreatology 2016;16:14-27.

28. Shi S, Ji S, Qin Y, et al. Metabolic tumor burden is associated with major oncogenomic alterations and serum tumor markers in patients with resected pancreatic cancer. Cancer Lett 2015;360:227-33.

29. Xu HX, Chen T, Wang WQ, et al. Metabolic tumour burden assessed by ${ }^{18} \mathrm{~F}$-FDG PET/CT associated with serum CA19-9 predicts pancreatic cancer outcome after resection. Eur J Nucl Med Mol Imaging 2014;41:1093-102.

30. van Manen L, Groen JV, Putter H, et al. Elevated CEA and CA19-9 serum levels independently predict advanced pancreatic cancer at diagnosis. Biomarkers 2020;25:186-93.

31. Ashfaq A, Pockaj BA, Gray RJ, et al. Nodal counts and lymph node ratio impact survival after distal pancreatectomy for pancreatic adenocarcinoma. J Gastrointest Surg 2014;18:1929-35.

32. Malleo G, Maggino L, Ferrone CR, et al. Number of Examined Lymph Nodes and Nodal Status Assessment in Distal Pancreatectomy for Body/Tail Ductal Adenocarcinoma. Ann Surg 2019;270:1138-46.

33. Zhang C, Dong S, Wang L, et al. Prognostic values of common clinical parameters in advanced pancreatic ductal adenocarcinoma: a large multicenter cohort study of ten years. Discov Med 2018;25:91-8.

34. Chen T, Zhang MG, Xu HX, et al. Preoperative serum

Cite this article as: Ge J, Li L, Ma Z, Jiang B, Yuan C, Wang H, Peng Y, Xiu D. A nomogram of preoperative predictors for occult metastasis in patients with PDAC during laparoscopic exploration. Gland Surg 2021;10(1):279-289. doi: 10.21037/gs20-605
CA125 levels predict the prognosis in hyperbilirubinemia patients with resectable pancreatic ductal adenocarcinoma. Medicine (Baltimore) 2015;94:e751.

35. Jiang K, Tan E, Sayegh Z, et al. Cancer Antigen 125 (CA125, MUC16) Protein Expression in the Diagnosis and Progression of Pancreatic Ductal Adenocarcinoma. Appl Immunohistochem Mol Morphol 2017;25:620-3.

36. Yang C, Cheng H, Luo G, et al. The metastasis status and tumor burden-associated CA125 level combined with the CD4/CD8 ratio predicts the prognosis of patients with advanced pancreatic cancer: A new scoring system. Eur J Surg Oncol 2017;43:2112-8.

37. Liu L, Xu HX, Wang WQ, et al. Serum CA125 is a novel predictive marker for pancreatic cancer metastasis and correlates with the metastasis-associated burden. Oncotarget 2016;7:5943-56.

38. Groot VP, Gemenetzis G, Blair AB, et al. Defining and Predicting Early Recurrence in 957 Patients With Resected Pancreatic Ductal Adenocarcinoma. Ann Surg 2019;269:1154-62.

39. Ferrone CR, Haas B, Tang L, et al. The influence of positive peritoneal cytology on survival in patients with pancreatic adenocarcinoma. J Gastrointest Surg 2006;10:1347-53.

40. Harada K, Murayama Y, Kubo H, et al. Photodynamic diagnosis of peritoneal metastasis in human pancreatic cancer using 5-aminolevulinic acid during staging laparoscopy. Oncol Lett 2018;16:821-8. 